\title{
Long-term cardiorenal efficacy of finerenone in patients with chronic kidney disease and type 2 diabetes
}

\author{
Mei Qiu^, Li-Min Zhao \\ Department of General Medicine, Shenzhen Longhua District Central Hospital, Shenzhen, China \\ Correspondence to: Mei Qiu. Department of General Medicine, Shenzhen Longhua District Central Hospital, Guanlan Avenue 187, Longhua District, \\ Shenzhen, China. Email: 13798214835@sina.cn.
}

Submitted Sep 11, 2021. Accepted for publication Sep 26, 2021.

doi: 10.21037/apm-21-2590

View this article at: https://dx.doi.org/10.21037/apm-21-2590

We read with great interest Fu et al.'s meta-analysis (1) recently published in the journal Annals of Palliative Medicine. In that meta-analysis (1) Fu and colleagues assessed the effects of finerenone on the short-term intermediate indicators such as urine albumin-to-creatinine ratio (UACR) and estimated glomerular filtration rate (eGFR) in patients with chronic kidney disease (CKD), as well as its safety. However, the authors (1) failed to assess the efficacy of finerenone on long-term renal and cardiovascular endpoints in CKD patients. Moreover, they (1) also failed to include the latest cardiorenal outcome trial (CROT) of finerenone, namely, the FIGARO-DKD trial (2) assessing cardiorenal outcomes with finerenone in patients with CKD and type 2 diabetes (T2D). Thus, we conducted a further meta-analysis by incorporating these two CROTs $(2,3)$ of finerenone [i.e., FIGARO-DKD (2) and FIDELIO-DKD (3)] to evaluate the long-term cardiorenal endpoints of finerenone in patients with T2D and CKD.

Both FIGARO-DKD (2) and FIDELIO-DKD (3) focused on comparing the cardiorenal outcomes of finerenone with those of placebo in patients with T2D and CKD. The former (2) involved 3,686 patients in the finerenone group and 3,666 patients in the placebo group, while the later (3) involved 2,833 patients in the finerenone group and 2,841 patients in the placebo group. Outcomes of interest for this meta-analysis were cardiovascular composite outcome (i.e., a composite of death from cardiovascular causes, nonfatal myocardial infarction, nonfatal stroke, or hospitalization for heart failure) and its components, kidney composite outcome (1) (i.e., a composite of kidney failure, a sustained decrease of at least $40 \%$ in the eGFR from baseline, or death from renal causes) and its components, kidney composite outcome (2) (i.e., a composite of kidney failure, a sustained decrease of at least $57 \%$ in the eGFR from baseline, or death from renal causes) and its components, death from any cause, and hospitalization for any cause. We extracted the study-level binary data (i.e., the numbers of events of interest and those of total subjects in each study group), and performed fixedeffects meta-analysis due to the lack of heterogeneity or low heterogeneity. Pooled effect size was presented as risk ratio (RR) and $95 \%$ confidence interval (CI). A P value of $<0.05$ denoted statistically significant difference. Meta-analyses were conducted in Stata/MP 16.0 (StataCorp LLC., College Station, TX, USA).

Figure 1 shows the results of meta-analysis on 14 longterm cardiorenal outcomes. Compared with placebo, finerenone reduced cardiovascular composite outcome (RR: $0.86,95 \%$ CI: $0.78-0.95, \mathrm{P}<0.01$; Figure $1 A$ ) by $14 \%$, hospitalization for heart failure (RR: $0.78,95 \%$ CI: $0.66-0.92$, $\mathrm{P}<0.01$; Figure $1 E$ ) by $22 \%$, kidney composite outcome (1) (RR: 0.83 , 95\% CI: $0.75-0.92, \mathrm{P}<0.01$; Figure $1 F$ ) by $17 \%$, end-stage kidney disease (RR: $0.80,95 \%$ CI: $0.64-0.99, \mathrm{P}=0.04$; Figure $1 H$ ) by $20 \%$, sustained decrease in eGFR of $<15 \mathrm{~mL} / \mathrm{min} / 1.73 \mathrm{~m}^{2}$ (RR: 0.81 , 95\% CI: $0.67-0.99, \mathrm{P}=0.04$; Figure 1 I) by $19 \%$, sustained $\geq 40 \%$

$\wedge$ ORCID: 0000-0001-5013-657X. 

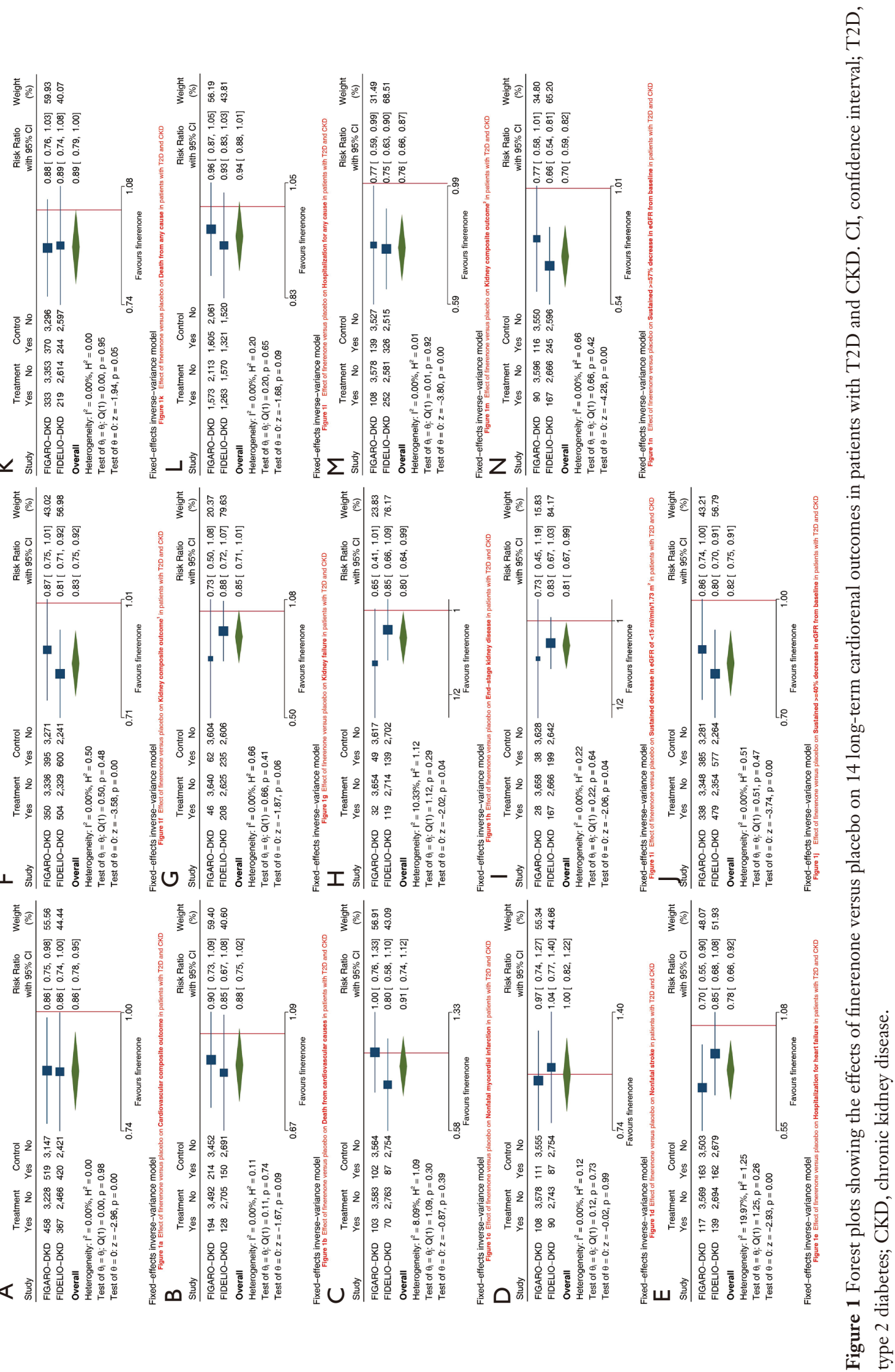
decrease in eGFR from baseline (RR: 0.82, 95\% CI: 0.75-0.91, $\mathrm{P}<0.01$; Figure 17) by $18 \%$, kidney composite outcome (2) (RR: $0.76,95 \%$ CI: $0.66-0.87, \mathrm{P}<0.01$; Figure $1 M$ ) by $24 \%$, and sustained $\geq 57 \%$ decrease in eGFR from baseline (RR: 0.70 , 95\% CI: $0.59-0.82, \mathrm{P}<0.01$; Figure $1 N$ ) by $30 \%$. Finerenone versus placebo showed the reduced trends in the risks of death from cardiovascular causes (RR: 0.88, 95\% CI: 0.75-1.02, $\mathrm{P}=0.09$; Figure $1 B$ ), kidney failure (RR: 0.85 , 95\% CI: 0.71-1.01, $\mathrm{P}=0.06$; Figure $1 G$ ), death from any cause (RR: 0.89, 95\% CI: $0.79-1.00, \mathrm{P}=0.05$; Figure $1 \mathrm{~K})$, and hospitalization for any cause (RR: 0.94, 95\% CI: 0.88-1.01, $\mathrm{P}=0.09$; Figure $1 L$ ). Finerenone versus placebo did not have significant effects on nonfatal myocardial infarction (RR: 0.91, 95\% CI: 0.74-1.12, $\mathrm{P}=0.39$; Figure $1 C$ ) and nonfatal stroke (RR: 1.00, 95\% CI: $0.82-1.22, \mathrm{P}=0.99$; Figure $1 D$ ).

Fu et al.'s meta-analysis (1) mainly revealed that finerenone versus placebo significantly reduced the UACR in patients with CKD. This suggests the benefits of finerenone on shortterm renal indicators, but does not mean that finerenone can provide long-term renal protective effects for CKD patients. Moreover, that meta-analysis (1) also failed to evaluate the long-term cardiovascular efficacy of finerenone versus placebo. On the contrary, our meta-analysis revealed that in CKD patients with T2D compared to placebo finerenone significantly reduced not only cardiovascular and renal composite outcomes but also individual cardiorenal outcomes including hospitalization for heart failure, end-stage kidney disease, and sustained decrease in eGFR. Moreover, in this meta-analysis finerenone versus placebo was also observed with the reduced trends in the risks of cardiovascular death and all-cause death in patients with T2D and CKD. Our findings suggest that finerenone should be recommended in patients with T2D and CKD to improve long-term cardiovascular and renal prognosis.

\section{Acknowledgments}

Funding: None.

Cite this article as: Qiu M, Zhao LM. Long-term cardiorenal efficacy of finerenone in patients with chronic kidney disease and type 2 diabetes. Ann Palliat Med 2021;10(10):11239-11241. doi: 10.21037/apm-21-2590

\section{Footnote}

Provenance and Peer Review: This article was a standard submission to the journal. The article did not undergo external peer review.

Conflicts of Interest: Both authors have completed the ICMJE uniform disclosure form (available at https://dx.doi. org/10.21037/apm-21-2590). The authors have no conflicts of interest to declare.

Ethical Statement: The authors are accountable for all aspects of the work in ensuring that questions related to the accuracy or integrity of any part of the work are appropriately investigated and resolved.

Open Access Statement: This is an Open Access article distributed in accordance with the Creative Commons Attribution-NonCommercial-NoDerivs 4.0 International License (CC BY-NC-ND 4.0), which permits the noncommercial replication and distribution of the article with the strict proviso that no changes or edits are made and the original work is properly cited (including links to both the formal publication through the relevant DOI and the license). See: https://creativecommons.org/licenses/by-nc-nd/4.0/.

\section{References}

1. Fu Z, Geng X, Chi K, et al. Efficacy and safety of finerenone in patients with chronic kidney disease: a systematic review with meta-analysis and trial sequential analysis. Ann Palliat Med 2021;10:7428-39.

2. Pitt B, Filippatos G, Agarwal R, et al. Cardiovascular Events with Finerenone in Kidney Disease and Type 2 Diabetes. N Engl J Med 2021. [Epub ahead of print]. doi:10.1056/NEJMoa2110956.

3. Bakris GL, Agarwal R, Anker SD, et al. Effect of Finerenone on Chronic Kidney Disease Outcomes in Type 2 Diabetes. N Engl J Med 2020;383:2219-29. 